\title{
Coupling digital and physical worlds in an AR magic show performance:
}

\author{
Anna Carreras, Carles Sora*
}

Institut Universitari de l'Audiovisual. Universitat Pompeu Fabra.

\begin{abstract}
"Magic for a Pixeloscope" is a one hour show conceived to be represented in a theater scenario that merges mixed and augmented reality (MR/AR) and full-body interaction with classical magic to create new tricks. The show was conceived by an interdisciplinary team composed by a magician, two interaction designers, a theater director and a stage designer. The magician uses custom based hardware and software to create new illusions which are a starting point to explore new language for magical expression. In this paper we introduce a conceptual framework used to inform the design of different tricks; we explore the design and production of some tricks included in the show and we describe the feedback received on the world premiere and some of the conclusions obtained.
\end{abstract}

KEYWORDS: Interaction design, magic, mixed reality, augmented reality, full-body interaction, magical expression, performance, interactive theater.

INDEX TeRms: H.5.1 [Multimedia Information Systems]: Artificial, augmented, and virtual realities; J.5. [Arts and Humanities]: Performing arts; J.7. [Computers in other systems]: Real time.

\section{INTRODUCTION}

Magicians have always been reinventing their shows and their magical language expression to fascinate the public with new tricks and illusions. With this purpose magic performances incorporate new technologies and inventions. However, in most of cases, the innovations are exhibited the mechanical and audiovisual field. Despite of the new possibilities, which the fields of MR/AR and interaction offer, very few magic shows incorporate this new media.

The usage of virtual reality (VR) and MR/AR technologies is growing in performing arts like dance-for example as in Mortal Engine spectacle [15] or in Apparition [1]-, theatre -as in Mesa di Vocce [13]- or even in opera -as in Medial Stage and Costume Design project [12]. On the contrary few magical performances incorporate MR/AR. Some magicians who are designing and using these new technologies as Marco Tempest [11] or Mago Julian [10] work together with artists as Zach Liberman [18] who can contribute with the interaction design part and the technological development.

To fill this gap we designed and produced a magical show entitled "Magic for a Pixeloscope". We incorporated interaction design into the show from scratch. We wanted to explore what

\footnotetext{
* Roc Boronat, 138. 08018 Barcelona. Spain.

\{anna.carrerass, carles.sora\}@upf.edu
}

this field can contribute to theatre. We believe that new digital technologies as MR/AR can offer new ways to understand the creation of a performance. Several remediations arose in theatre during its history contributing with new theatre approaches [17]. Each remediation caused a new way to understand the nature of theatre, the way to represent it and the role of actors on stage [2].

Indeed, nowadays, the performer can control most of the media and mechanic devices trough his body and actions. His body can be understood as the control room where all the spectacular elements are derived and controlled [3]. Even if audience takes part of the show the new digital performance can control the advent of actions of the participant.

The role of a magician on stage is to control the tricks' mechanics and his actions constantly in order to create amazing illusions. This project pushes towards the usage of MR/AR and interactive technologies on stage exploring how to provoke new experiences in the audience, promoting the analysis and understanding of this new communication media and the study of its intrinsic characteristics [8].

We believe that the experience we develop could not be achieved on stage using any other media.

\section{Conceptualizing Mr/AR IN terms OF MAgical EFFECTS}

Previous research into conceptualizing mixed realities focused on moving from one physical place to another virtual one and vice versa via boundaries, which transparently connect separate, non-overlaid physical and virtual spaces. For example, Milgram [14] classifies mixed realities according a 'continuum' characterized by two extremes - the physical environment on one side, and the other virtual environment on the other. One of the main conceptualizing ideas for the show was to explore this relation between the real and unreal, i.e. the relation between the physical and the digital or virtual; and how the relation between two worlds and the boundaries between them can be used to create a magical effect in the audience.

Koleva et al [4][5] describe the properties of a 'traversable' interface connecting two worlds, as something which "gives the illusion of joining physical and virtual worlds together and that users can physically cross from one to the other". The relation between physical world and digital one (the term 'physical' used to allude to actions or effects which do not involve virtual/digitally-enhanced artifacts) is reached through interfaces that require that the audience believe the illusion, while the magician might be aware of the mechanisms involved. This idea challenges traditional approaches to interface design that have focused on the experience of the direct participant neglecting the experience of observers [5]. Hence, new tricks are designed for the show mixing magic and MR/AR and new interfaces are developed for the magician to perform the trick and create the desired illusion in the audience.

A magician can make a coin appear in the air, out of nothing, and this effect will have a high grade of illusion in the audience. 
By using interactive technologies, the magician can also make the coin appear inside a screen just touching the screen surface. However, as the audience is nowadays quite used to touch screens interfaces - that can have some magical flavor when experienced -, this effect will have a low grade of illusion on the public if a magician is performing it on stage.

On the contrary, we believe that coupling both effects and worlds will have a higher illusion impact, as proposed in [6] for the performance climax. For example, the magician could grasp a coin that is placed inside the screen making it disappear from there, the digital world, and bringing it to the physical world, making the coin appear on his hand. In this case the magical effect is reached by crossing and breaking the boundaries between the physical world and the digital one, coupling the two extremes of the mixed realities continuum.

\section{Magical efFects}

Interaction and MR/AR are used to go from one world to the other by using different transforms [16]. We will analyze four different combinations, i.e. transforms, for coupling these two worlds:

Physical world action -> Physical world effect $(\mathrm{PPt})$

Physical world action -> Digital world effect (PDt)

Digital world action $->$ Digital world effect (DDt)

Digital world action -> Physical world effect (DPt)

The design and construction of these transforms from one world to the other and vice versa implies that a magical effect, could exist, in the physical world, in digital world or in both.

To decide which kind of tricks could be suitable to bring physical and virtual worlds together achieving a high magical illusion 1) we describe major magic effects categories, which already exist on the physical world; 2) we analyze if the magical effect can be also performed in the digital world, posing an example of each category; 3 ) we evaluate the possibility to couple the digital effect with the physical world (low, medium and high); and 4) we estimate which grade of illusion can the digital effect afford for each transform (low, medium and high), see Table 1.

Table 1. Magical effects' categories, a digital example, the possibility to couple it with the physical world and the estimated grade of illusion that the transformation produces in the audience.

\begin{tabular}{|c|c|c|c|}
\hline Magical effect category & Digital world effect example & $\begin{array}{l}\text { Possibility of coupling } \\
\text { digital with physical world }\end{array}$ & $\begin{array}{l}\text { Estimated grade of } \\
\text { illusion of each transform } \\
\text { type }\end{array}$ \\
\hline $\begin{array}{l}\text { Production: the magician produces } \\
\text { something from nothing. }\end{array}$ & $\begin{array}{l}\text { The magician makes a coin } \\
\text { appear on screen just touching the } \\
\text { screen surface }\end{array}$ & High & $\begin{array}{l}\text { PPt: High } \\
\text { PDt: Medium } \\
\text { DDt: Low } \\
\text { DPt: Medium }\end{array}$ \\
\hline $\begin{array}{l}\text { Vanishing: the magician makes } \\
\text { something disappear }\end{array}$ & $\begin{array}{l}\text { The magician makes a coin } \\
\text { placed on a screen disappear just } \\
\text { touching it or snapping his fingers }\end{array}$ & High & $\begin{array}{l}\text { PPt: High } \\
\text { PDt: Medium } \\
\text { DDt: Low } \\
\text { DPt: High }\end{array}$ \\
\hline $\begin{array}{l}\text { Transformation: the magician } \\
\text { transforms something from one state } \\
\text { into another. }\end{array}$ & $\begin{array}{l}\text { The magician can manipulate } \\
\text { in real time an image of an object } \\
\text { by stretching, deforming, etc. it }\end{array}$ & Medium & $\begin{array}{l}\text { PPt: Medium } \\
\text { PDt: Medium } \\
\text { DDt: Low } \\
\text { DPt: High }\end{array}$ \\
\hline $\begin{array}{l}\text { Restoration: the magician destroys } \\
\text { an object, then restores it back to its } \\
\text { original state. }\end{array}$ & $\begin{array}{l}\text { The magician can on the screen } \\
\text { put together the parts of a broken } \\
\text { object and when together restore } \\
\text { the object by snapping his fingers }\end{array}$ & High & $\begin{array}{l}\text { PPt: High } \\
\text { PDt: Low } \\
\text { DDt: Medium } \\
\text { DPt: High }\end{array}$ \\
\hline $\begin{array}{l}\text { Teleportation: the magician causes } \\
\text { something to move from one place to } \\
\text { another. }\end{array}$ & $\begin{array}{l}\text { The magician makes an object } \\
\text { disappear from one side of the } \\
\text { screen appearing in another } \\
\text { corner of the image }\end{array}$ & $\begin{array}{l}\text { Low (if not coupled with } \\
\text { Production and Vanishing) }\end{array}$ & $\begin{array}{l}\text { PPt: High } \\
\text { PDt: Medium } \\
\text { DDt: Medium } \\
\text { DPt: Medium }\end{array}$ \\
\hline $\begin{array}{l}\text { Prediction: the magician predicts } \\
\text { the choice of a spectator, or the } \\
\text { outcome of an event under seemingly } \\
\text { impossible circumstances. }\end{array}$ & - & - & - \\
\hline $\begin{array}{l}\text { Levitation: the magician defies } \\
\text { gravity, either by making something } \\
\text { float in the air, or with the aid of } \\
\text { another object (suspension). }\end{array}$ & $\begin{array}{l}\text { The magician makes an object } \\
\text { float over his hand controlling its } \\
\text { position in real time }\end{array}$ & $\begin{array}{l}\text { (both worlds are already } \\
\text { connected in this case as the } \\
\text { controlling object is in the } \\
\text { physical world and the } \\
\text { controlled object in the } \\
\text { digital) }\end{array}$ & $\begin{array}{l}\text { PPt: High } \\
\text { PDt: Medium } \\
\text { DDt: Low } \\
\text { DPt: Medium }\end{array}$ \\
\hline
\end{tabular}

\subsection{Two examples}

In order to clarify how to design and create a trick by breaking the boundaries of the physical and digital world we will describe two examples.

For the case of a transformation effect doing a digital action and having a result in the physical world (Dpt), imagine that the magician proposes a volunteer to choose photography or a card. After the choice is done the card is placed inside the digital space where the volunteer is asked to draw something on the photography, following the narrative of the show. After the photo is changed the magician returns the card, via a production (Dpt), 
to the physical world, where the changes made in the physical plane remain, and the real photography has also been drawn.

We detail another example: a restoration effect. We can imagine the magician asking a volunteer to give him her mobile phone. By chance this mobile phone is destroyed on stage, breaking into several pieces. But, the magician wants to repair the damage done and promises the volunteer to restore her mobile phone. The magician, by a production (Pdt), introduces all the pieces inside a screen where the volunteer is asked to glue them together by touching the screen and dragging the pieces inside a virtual box. Once the pieces are glued the magician, via a Dpt production and a Ddt vanishing effect, gets back the volunteer's mobile phone completely restored.

\section{MAGIC FOR A PIXELOSCOPE}

"Magic for a Pixeloscope" is a one hour magical show that includes some of the transforms described above in different routines - a collection of tricks, actions and procedures that are followed regularly by a magician to perform a bigger illusion effect -, see Table 1 .

The purpose of the project was to explore new languages for illusion and magical effects by:

Putting together an interdisciplinary group of people discussing about new ways to create magical effects and illusions;

Introducing $\mathrm{MR} / \mathrm{AR}$ and full-body interaction technologies to achieve these illusions;

Designing and producing a magic performance with some of the analyzed transformations.

Verifying if the estimated grade of illusion for the transformations developed for the show was the one produced on the public.

\subsection{Installation and Technology}

Custom based hardware and software was developed to capture and process the magician and audience volunteers' gestures and behaviors which were used to create digitally generated real-time stimuli. We developed two different interfaces which permitted the performance of the designed new tricks:

An interactive table for close magic tricks where magician hand gestures and cards were captured by a camera, see Figure 1. Camera was placed on the top of the table to show a clear zenith-view of the magician manipulating cards. The live video capture was shown on a big screen and the real-time effects, controlled by the magician, augmented the video image. Volunteers and the magician manipulate and create the effects in real time.

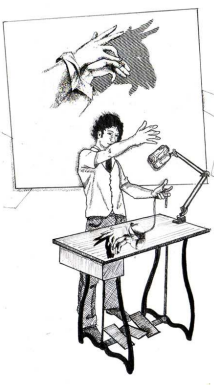

a)

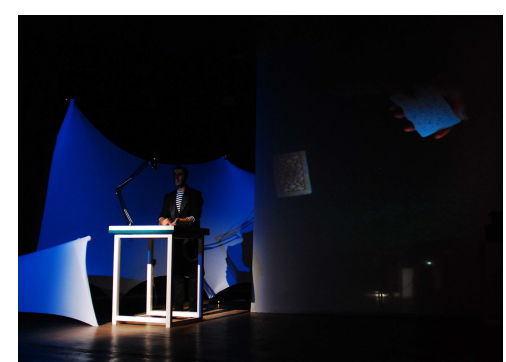

b)

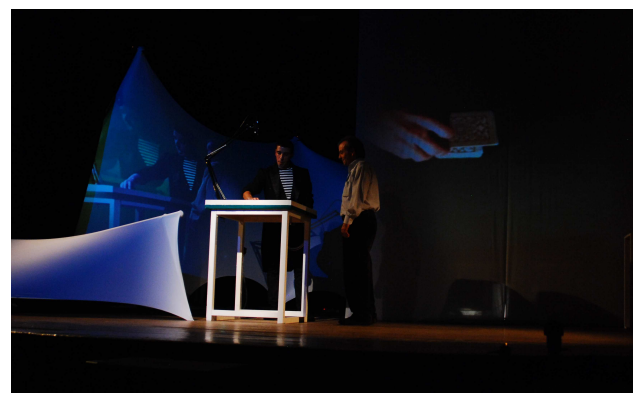

c)

Figure 1. Interactive table with a camera for augmenting cards and magician hands; a) initial design, b) interface on stage, c) the magician with a volunteer.

A big interactive retro projected screen. In front of it movement, behaviors, position and full-body gestures were captured, processed and used to interact with projected elements in a 1:1 scale, see Figure 2. The fullbody interaction is not mediated through users' silhouette, as on Myron Krueger's Videoplace [7]. In our setting, the full-body interaction technique is used for a first person interaction without any outline of the users' silhouette or any avatar, enhancing the illusion of controlling the digital world and the magical effect. For the projection to be seen well enough the screen needs to be dark. However for the computer vision application to work properly, illumination is needed. As the projection has to be visible to the public, the computer vision needs to operate in a different invisible spectrum such as infrared. Infrared lights were placed behind the screen and used to flood the screen. As the background is highly illuminated and the participants block the light arriving to the camera, they can be detected by the system robustly under different illumination scenery conditions.

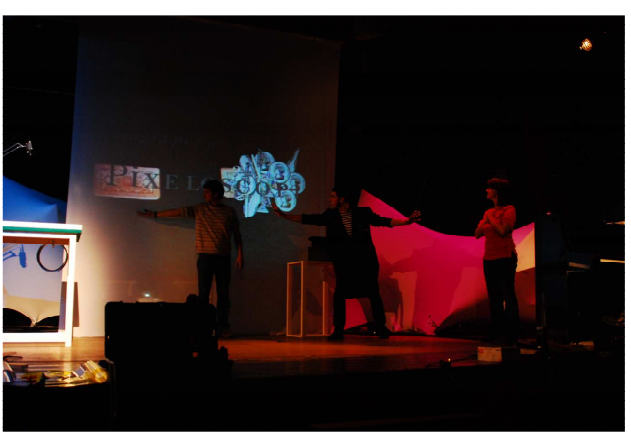

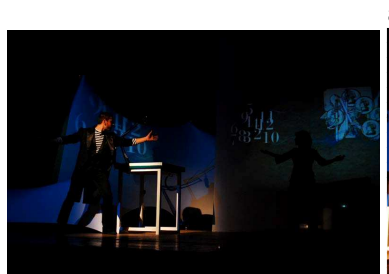

b)

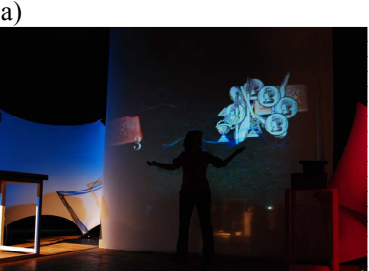

c)
Figure 2. Interactive screen for full-body interaction: a) an audience volunteer stands in front of the screen and extends his arm to control and augment with digital numbers and suits the cards on the table, b) and c) moving her arms another volunteer will get attached the correct number and suit of a selected card predicting it on the digital world. 


\subsection{A restoration routine for "Magic for a Pixeloscope"}

As mentioned above the show include a trick were the magician destroyed on stage, physical world, a volunteer's mobile phone, breaking it into several pieces. Afterwards all the pieces were placed inside a screen, the digital world, where the volunteer recomposed the mobile by dragging the pieces and putting them inside a virtual box that appeared inside the screen, see Figure 3.

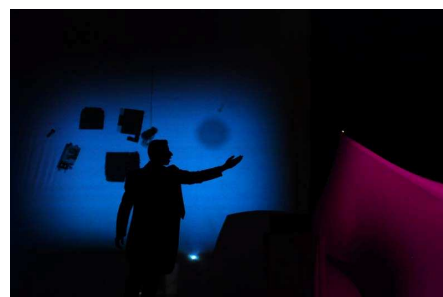

a)

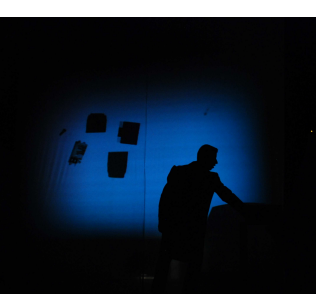

b)
Figure 3. The magician in front of the big screen with the broken mobile pieces on the left: a) he shows to a volunteer how to capture them, b) and he put the pieces inside the virtual box, on the right below.

The magician finally got back the volunteer's mobile phone completely restored to the real world, making it appear inside a box on stage, see Figure 3. This routine included two different magical effects and three different transformations: a production with a Pdt transformation to introduce the mobile pieces from physical world into the screen; a Dpt production and a Ddt vanishing effect coupled to get the reconstructed mobile phone out of the screen and appearing inside - which can be understood as a teleportation from digital world to physical world -, see Figure 4.

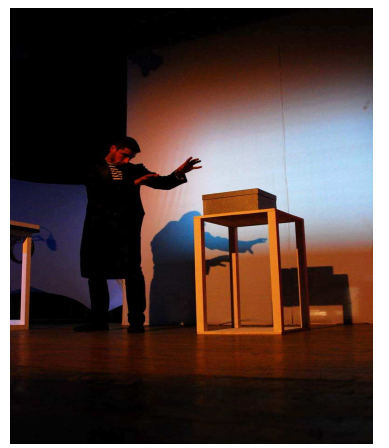

Figure 4. The magician teletransported a mobile phone from the screen to the physical box on stage via a Dpt production and a Ddt vanishing.

\section{CONCLUSIONS}

The world premiere was the 19th December 2008 in Can Felipa's theater, Barcelona - a 5 minutes video can be found in [9] -. 104 spectators assisted to the show, aged from 6 years old to 72 years old. Among the audience there were magicians, interaction designers and plain public. We received an amazingly good feedback from them. We collected the feedback through interviews from observers and three different volunteers that participated in the show experiencing the interactions and tricks proposed.

The main lessons we learned were:

A good way to achieve illusion by mixing MR/AR technologies with classical magic is by breaking the boundaries between physical and digital worlds through the usage of transforms.
Not all the transforms could achieve the same grade of illusion in the audience. The restoration routine detailed above achieved a high degree of illusion and was the most liked by the audience. To achieve it three different transforms were concatenated and coupled.

The magician performance, actions and attitude contributes to the understanding of transforms and audience fascination.

"Magic for a Pixeloscope" will evolve whit the lessons learned during the initial performances. We will design new tricks and effects and will test their grade of illusion. The established framework is a starting point to understand the possibilities that new technologies like MR/AR offer to magic and performing arts, an umbrella to contribute with new shows to the evolution and modernization of these fields.

\section{ACKNOWLEDGMENTS}

Thanks to Magic Jaïr, magician, Martí Baltà, set designer and Ferran Utzet, stage director. We also thank Fundacio Phonos for their support to produce "Magic for a Pixeloscope" and Can Felipa to let us represent the show in such a magical old building.

\section{References}

[1] Apparition project: http://www.exile.at/apparition/

[2] G. Giannachi. Virtual Theatres, an introduction. Routledge. Canada. 2004.

[3] O. Grau. Virtual Art: From Illusion to Immersion. Leonardo Books. The MIT Press. October 2004

[4] B. Koleva, S. Benford, and C. Greenhalgh, The Properties of Mixed Reality Boundaries, Proceedings of ECSCW'99 September 1999.

[5] B. Koleva, H.M. Schnadelbach, S. Benford and C.M. Greenhalgh. Traversable interfaces between real and virtual worlds. Proceedings of Conference on Human Factors in Computer Systems CHI'00 (Netherlands, April 2000) Proc. pages 233-240, ACM Press, 2000.

[6] B. Koleva, I. Taylor, S. Benford, M. Fraser, C.M. Greeenhalgh, H.M. Schnadelbach, D. vom Lehn, C. Heath, J. Row-Fatt, M. Adams. Orchestrating a mixed reality performance. Proceedings of Conference on Human Factors in Computer Systems CHI'01. Proc. pages 38-45, ACM Press, 2001.

[7] M. Krueger. VIDEOPLACE and the Interface of the Future. Addison-Wesley Professional. January 1990.

[8] B. Laurel. Computers as Theatre. Addison-Wesley. 1992.

[9] "Magic for a Pixeloscope" video project: http://vimeo.com/3326652

[10] Mago Julian web page: http://thesystemis.com/opensourcery/

[11] Marco Tempest web page: http://www.marcotempest.com/screen/Public_News_Detail/language /en/newsid/News_1239939382909

[12] Medial Stage project: http://www.artcom.de/index.php?option=com_acprojects\&page $=6 \& \mathrm{i}$ $\mathrm{d}=29$ \&Itemid $=113$ \&details $=0$ \&lang $=$ en

[13] Mesa di Voce project: http://www.tmema.org/messa/messa.html\#overview

[14] P. Milgram and F. Kishino. A Taxonomy of Mixed Reality Visual Displays. IEICE Transactions on Information Systems. vol E77-D (12). December 1994.

[15] Mortal Engine project: http://www.chunkymove.com/OurWorks/Current-Productions/Mortal-Engine.aspx

[16] Y. Rogers, M. Scaife, S. Gabrielli, H. Smith and E. Harris. A conceptual framework for mixed reality environments: designing novel learning activities for young children. Presence: Teleoper. Virtual Environ., vol. 11, no. 6, pages. 677-686. December 2002.

[17] C. Sora Seven reformulations for a interactive dramaturgy. iEnter'08. Interactive Entertainment Workshop. Barcelona, 2008.

[18] Zach Liberman web page: http://www.thesystemis.com/ 